NBI-HE-92-41

June 1992

\title{
VANISHING OF THE COSMOLOGICAL CONSTANT IN STABILIZED QUANTUM GRAVITY四
}

\author{
J. Greensite P \\ The Niels Bohr Institute \\ Blegdamsvej 17 \\ DK-2100 Copenhagen Ø, Denmark
}

\begin{abstract}
It is shown that the probability distribution $P(\lambda)$ for the effective cosmological constant is sharply peaked at $\lambda=0$ in stochastic (or "fifth-time") stabilized quantum gravity. The effect is similar to the Baum-Hawking mechanism, except that it comes about due to quantum fluctuations, rather than as a zeroth-order (in $\hbar$ ) semiclassical effect.
\end{abstract}

\footnotetext{
${ }^{1}$ Supported by the U.S. Department of Energy under Grant No. DE-FG03-92ER40711.

${ }^{2}$ Permanent address: Physics and Astronomy Dept., San Francisco State University, San Francisco, CA 94132 USA.
} 
Very simple and elegant arguments for the vanishing of the cosmological constant were advanced some time ago, in the context of Euclidean quantum gravity, by Baum [1], Hawking [2], and Coleman [3]. The Baum-Hawking argument, in particular, can be summarized in a few lines: The effective cosmological constant $\lambda$ is the sum of the bare cosmological constant $\lambda_{0}$ plus a contribution from the stressenergy of the non-gravitational fields, and it is assumed that the range of possible $\lambda$ includes the value $\lambda=0$. Integrating over the gravitational field then gives a probability distribution for $\lambda$,

$$
P(\lambda)=\int D g \exp \left[-\int d^{4} x \sqrt{g}\left[-\frac{1}{16 \pi G} R+\lambda\right]\right]
$$

and the semiclassical evaluation of this expression gives

$$
P(\lambda) \approx \exp \left[\frac{3}{8 G^{2} \lambda}\right]
$$

which is peaked at infinity as $\lambda \rightarrow 0^{+}$. This infinite peak in the probability distribution at $\lambda=0^{+}$is the Baum-Hawking explanation for the vanishing of the cosmological constant. Coleman's argument [3], which appeals to wormhole effects, is a related idea, and gives at the semiclassical level a double exponential distribution

$$
P(\lambda) \approx \exp \left[\exp \left[\frac{3}{8 G^{2} \lambda}\right]\right]
$$

Unfortunately there is a strong objection to the Baum-Hawking-Coleman arguments; namely, that these arguments rely on (and suffer from) the fact that the Euclidean Einstein-Hilbert action is unbounded from below. At the classical level, the action at the stationary point $\delta S=0$ is $S=-3 /\left(8 G^{2} \lambda\right)$, which leads to the semiclassical distributions (2) and (3) above, but in fact the action can be made arbitrarily negative, due to the well-known "wrong-sign" of the kinetic term of the metric conformal factor. This unboundedness of the action from below means that the functional integral in (1), and the Euclidean quantum theory based on it, are essentially meaningless. If one nevertheless attempts to define the integral over the conformal factor by a contour rotation [4], then the unboundedness problem is simply shifted to the matter Lagrangian [5]. Even in pure gravity, the conformal rotation introduces a complex phase $(-i)^{D+2}$ in front of the second exponential of eq. (3), which ruins the Coleman argument in $\mathrm{D}=4$ dimensions [6]. Finally, deformation of a field integration contour into the complex plane tends to generate complex expectation values of physical quantities at the non-perturbative level; this is known to occur in matrix models for $2 \mathrm{D}$ gravity [7].

However, there exists a general method, grounded in stochastic quantization, for defining the Euclidean quantum theory of any action unbounded from below. 
This is the stochastic stabilization, or "fifth-time action" method, introduced in ref. [8], which has the property of stabilizing the action while preserving the classical limit and, if the bottomless action is stable at zeroth order, also preserving the naive perturbative expansion to all orders in any coupling constant. The method has been applied to $\mathrm{D}=0$ matrix models representing $2 \mathrm{D}$ gravity in ref. [9], and to the Einstein-Hilbert and Einstein-Cartan actions of 4D gravity in ref. 10] and [11. Very recently there has also been some been work on the cosmological constant issue in stochastic stabilized quantum gravity. Carlini and Martellini [12] have studied a minisuperspace approximation of the stabilized Einstein-Hilbert action, while the author, in ref. [11], has carried out a Monte Carlo calculation of the stabilized, latticized, Einstein-Cartan theory. In both cases there appears to be evidence for a peaking of the probability distribution around $\lambda=0^{+}$. These results motivate the one-loop calculation presented here.

It was first noted by Giveon et. al. [13] (reviewed in more detail in ref. [11), that the fifth-time action method of ref. [8] is completely equivalent to Langevin evolution, i.e. stochastic quantization, between fixed non-singular (but otherwise arbitrary) initial and final field configurations in the fictitious time. The precise prescription for this "stochastic stabilization" is as follows: Let $g^{M}$ be the fields, $G_{M N}$ the supermetric, and $E_{M}^{A}$ the supervielbein, of a given field theory. Evolution in the fictitious time $t_{5}$ is given by the Langevin equation

$$
\partial_{5} g^{M}\left(\mathbf{x}, t_{5}\right)=-G^{M N} \frac{\delta S}{\delta g^{N}}+E_{A}^{M} \eta^{A}
$$

starting from an initial configuration at $t_{5}=-T$

$$
g^{M}(\mathbf{x},-T)=g_{i}^{M}(\mathbf{x})
$$

Then the expectation value $\left\langle Q>\right.$ of any operator $Q\left[g^{M}(\mathbf{x})\right]$ in the stabilized Euclidean theory is given by

$$
\begin{aligned}
<Q>= & \lim _{T \rightarrow \infty} \frac{1}{Z_{5}} \int D \eta\left(\mathbf{x},-T<t_{5}<T\right) Q\left[g^{M}(\mathbf{x}, 0)\right] \delta\left[g^{M}(\mathbf{x}, T)-g_{f}^{M}(\mathbf{x})\right] \\
& \times \exp \left[-\int_{-T}^{T} d^{5} x \eta^{A} \eta_{A} / 4 \hbar\right]
\end{aligned}
$$

The only difference between the prescription (6), and ordinary stochastic quantization, is the delta function enforcing the final state constraint $g^{M}(\mathbf{x}, T)=g_{f}^{M}(\mathbf{x})$. This constraint prevents the system from running away to a singular configuration as $T \rightarrow \infty$, thereby stabilizing the theory. It can be shown in general that in the $T \rightarrow \infty$ limit, $<Q>$ is independent of the choice of initial and final configurations 
$g_{i}^{M}$ and $g_{f}^{M}$.p By a change of variables (c.f. [11), eq. (6) can be converted into the form

$$
\begin{aligned}
<Q> & =\frac{1}{Z_{5}} \int D g^{M}\left(\mathbf{x}, t_{5}\right) \sqrt{G} Q\left[g^{M}(\mathbf{x}, 0)\right] e^{-S_{5} / \hbar} \\
S_{5} & =\int d^{5} x\left[\frac{1}{4} G_{M N} \partial_{5} g^{M} \partial_{5} g^{N}+\frac{1}{4} G^{M N} \frac{\delta S}{\delta g^{M}} \frac{\delta S}{\delta g^{N}}-\frac{\hbar}{2} G^{M N} \frac{\delta^{2} S}{\delta g^{M} \delta g^{N}}\right]
\end{aligned}
$$

where $S_{5}$ is the "fifth-time" action.

Applying stochastic stabilization to Einstein-Cartan gravity

$$
S_{E C}=\int \epsilon_{a b c d}\left[-\frac{1}{4 \kappa^{2}} e^{a} \wedge e^{b} \wedge\left(d \omega^{c d}+\omega^{c f} \wedge \omega^{f d}\right)+\lambda_{0} \int d^{4} x \operatorname{det}(e)\right.
$$

leads to the stabilized formulation

$$
<Q[e, \omega]>=\frac{1}{Z_{5}} \int D e D \omega \sqrt{G} Q[e(\mathbf{x}, 0), \omega(\mathbf{x}, 0)] e^{-S_{5} / \hbar}
$$

where

$$
\begin{aligned}
S_{5}= & \frac{1}{4} \int d^{5} x \sqrt{g}\left[\frac{1}{\kappa^{2}} g^{\mu \nu}\left(\partial_{5} e_{\mu}^{a} \partial_{5} e_{\nu}^{a}+\hbar \partial_{5} \omega_{\mu}^{a b} \partial_{5} \omega_{\nu}^{a b}\right)\right. \\
& +4\left(\frac{1}{\kappa^{2}} R_{\mu}^{a} R_{\nu}^{a} g^{\mu \nu}-\lambda_{0} R+\kappa^{2} \lambda_{0}^{2}\right) \\
& \left.+\frac{1}{\kappa^{4} \hbar} T_{\mu \nu}^{a} T_{\rho \sigma}^{b}\left(\delta_{a b} g^{\mu \rho}+2 e_{a}^{\mu} e_{b}^{\rho}\right) g^{\nu \sigma}\right]
\end{aligned}
$$

is the 5-th time action and

$$
\begin{aligned}
& R=d \omega+\omega \wedge \omega \\
& T=d e+\omega \wedge e
\end{aligned}
$$

are the curvature and torsion two-forms respectively. The root determinant of the $e-\omega$ supermetric is

$$
\sqrt{G}=\sqrt{G_{e}} \sqrt{G_{\omega}} \propto \prod_{\mathbf{x}, t_{5}} \operatorname{det}^{10}(e)
$$

where $G_{e}$ and $G_{\omega}$ denote the separate tetrad and spin-connection supermetrics. The range of functional integration is restricted to tetrads representing compact 4-manifolds, at each $t_{5}$.

The stabilized theory of Euclidean gravity, defined above, is invariant with respect to $\mathrm{D}=4$ diffeomorphisms, yields the usual Einstein field equations in its classical limit, contains no higher-derivative terms, and appears to be reflection-positive

\footnotetext{
${ }^{3}$ In particular, for ordinary bounded actions, the prescription (6) is equivalent to the usual formulations.
} 
(at least in certain lattice versions) across the ordinary time $\left(x_{4}\right)$ axis [10]. It can also be expressed in terms of an effective four-dimensional action

$$
\begin{aligned}
& \exp \left\{-S_{\text {eff }}\left[e^{\prime}(\mathbf{x}), \omega^{\prime}(\mathbf{x})\right] / \hbar\right\} \\
& \quad=\int D e\left(\mathbf{x}, t_{5}\right) D \omega\left(\mathbf{x}, t_{5}\right) \sqrt{G} \delta\left[e(\mathbf{x}, 0)-e^{\prime}(\mathbf{x})\right] \delta\left[\omega(\mathbf{x}, 0)-\omega^{\prime}(\mathbf{x})\right] e^{-S_{5} / \hbar}
\end{aligned}
$$

which can be calculated perturbatively, as discussed in [10].

We now consider coupling stabilized gravity to non-gravitational fields, denoted collectively by $\phi$. Treating $\phi(\mathbf{x})$ as a $t_{5}$-independent source leads to the stabilized four-dimensional action

$$
\begin{aligned}
& \exp \left\{-S_{\text {eff }}\left[\phi(\mathbf{x}), e^{\prime}(\mathbf{x}), \omega^{\prime}(\mathbf{x})\right] / \hbar\right\} \\
& \quad=\int D e\left(\mathbf{x}, t_{5}\right) D \omega\left(\mathbf{x}, t_{5}\right) \sqrt{G} \delta\left[e(\mathbf{x}, 0)-e^{\prime}(\mathbf{x})\right] \delta\left[\omega(\mathbf{x}, 0)-\omega^{\prime}(\mathbf{x})\right] e^{-S_{5} / \hbar}
\end{aligned}
$$

and expectation values

$$
<Q>=\frac{1}{Z} \int D \phi(\mathbf{x}) \int D e\left(\mathbf{x}, t_{5}\right) D \omega\left(\mathbf{x}, t_{5}\right) \sqrt{G} Q[\phi(\mathbf{x}), e(\mathbf{x}, 0), \omega(\mathbf{x}, 0)] e^{-S_{5} / \hbar}
$$

where

$$
\begin{aligned}
S_{5}= & \frac{1}{4} \int d^{5} x \sqrt{g}\left[\frac{1}{\kappa^{2}} g^{\mu \nu}\left(\partial_{5} e_{\mu}^{a} \partial_{5} e_{\nu}^{a}+\hbar \partial_{5} \omega_{\mu}^{a b} \partial_{5} \omega_{\nu}^{a b}\right)\right. \\
& +\frac{\kappa^{2}}{4} g_{\alpha \beta}\left[\frac{2}{\kappa^{2}}\left(R_{c}^{\alpha}-\frac{1}{2} R e_{c}^{\alpha}\right)+\lambda_{0} e_{c}^{\alpha}+\mathcal{T}_{c}^{\alpha}\right]\left[\frac{2}{\kappa^{2}}\left(R_{c}^{\beta}-\frac{1}{2} R e_{c}^{\beta}\right)+\lambda_{0} e_{c}^{\beta}+\mathcal{T}_{c}^{\beta}\right] \\
& \left.+\frac{1}{\kappa^{4} \hbar} T_{\mu \nu}^{a} T_{\rho \sigma}^{b}\left(\delta_{a b} g^{\mu \rho}+2 e_{a}^{\mu} e_{b}^{\rho}\right) g^{\nu \sigma}\right]
\end{aligned}
$$

and $\mathcal{T}_{\mu \nu}(\phi(\mathbf{x}))$ is the stress-energy tensor for the non-gravitational $\phi$ fields.

In principle one could include the $\phi$ fields, along with the tetrad and spinconnection, among the $t_{5}$-dependent $g^{M}$ fields of the Langevin equation (田). This is not done here for two reasons: first, in the classical $\hbar \rightarrow 0$ limit, (16) implies the Einstein field equations,

$$
R_{\mu \nu}-\frac{1}{2} R g_{\mu \nu}+\frac{\kappa^{2}}{2} \lambda_{0} g_{\mu \nu}=-\frac{\kappa^{2}}{2} \mathcal{T}_{\mu \nu}
$$

and the Einstein field equations in turn imply the classical equations of motion of the $\phi$ fields [14]. Stabilizing the (already stable) non-gravitational fields via the Langevin equation seems redundant. Second and more importantly, if the $\phi$ are dynamic in $t_{5}$, then the fifth-time action $S_{5}$ contains higher-derivative terms coming from $(\delta S / \delta \phi)^{2}$, which threaten reflection-positivity for reflections across $x_{4}$. This 
problem is avoided if the non-gravitational fields are treated as $t_{5}$-independent, as above.

From (15) we see that integration over the tetrad $e=\bar{e}+\delta e$ and spin connection $\omega=\bar{\omega}+\delta \omega$ produces a probability measure for the non-gravitational fields on a ( $\phi$-dependent) background manifold $(\bar{e}, \bar{\omega})$, i.e.

$$
P[\phi(\mathbf{x})]=\frac{1}{Z} e^{-S_{e f f}[\phi] / \hbar}=\frac{1}{Z} \int D e\left(\mathbf{x}, t_{5}\right) D \omega\left(\mathbf{x}, t_{5}\right) \sqrt{G} e^{-S_{5}[e, \omega, \phi] / \hbar}
$$

Let us consider, in particular, non-gravitational field configurations, such as $\phi=$ const., in which the stress-energy is proportional to the metric tensor

$$
\mathcal{T}_{\mu \nu}=\tau g_{\mu \nu}
$$

Then the stress-energy can be combined with $\lambda_{0}$ to produce an effective cosmological constant

$$
\lambda=\tau+\lambda_{0}
$$

with a probability distribution

$$
P[\lambda]=\frac{1}{Z} \int D e\left(\mathbf{x}, t_{5}\right) D \omega\left(\mathbf{x}, t_{5}\right) \sqrt{G} e^{-S_{5}[e, \omega, \lambda] / \hbar}
$$

where $S_{5}[e, \omega, \lambda]$ is identical to the $S_{5}$ in eq. (10), with the replacement $\lambda_{0} \rightarrow \lambda$.

The action in (21) is bounded from below, and it is now meaningful to ask whether the probability $P(\lambda)$ is peaked as $\lambda \rightarrow 0^{+}$. For a given $\lambda>0$, the integral is to be evaluated by expansion around

$$
\begin{aligned}
e_{\mu}^{a}\left(\mathbf{x}, t_{5}\right) & =\bar{e}_{\mu}^{a}(\mathbf{x})+\delta e_{\mu}^{a}\left(\mathbf{x}, t_{5}\right) \\
\omega_{\mu}^{a b}\left(\mathbf{x}, t_{5}\right) & =\bar{\omega}_{\mu}^{a b}(e)+\kappa^{2} \sqrt{\hbar} \Omega_{\mu}^{a b}\left(\mathbf{x}, t_{5}\right)
\end{aligned}
$$

where $\bar{e}(\mathbf{x})$ is a tetrad for the 4 -sphere of radius

$$
r=\left(\frac{6}{\kappa^{2} \lambda}\right)^{1 / 2}
$$

and $\bar{\omega}(e)$ is the zero-torsion spin-connection

$$
\bar{\omega}_{\mu}^{a b}=\frac{1}{2} e^{\nu a}\left(\partial_{\mu} e_{\nu}^{b}-\partial_{\nu} e_{\mu}^{b}\right)-\frac{1}{2} e^{\nu b}\left(\partial_{\mu} e_{\nu}^{a}-\partial_{\nu} e_{\mu}^{a}\right)-\frac{1}{2} e^{\rho a} e^{\sigma b}\left(\partial_{\rho} e_{\sigma c}-\partial_{\sigma} e_{\rho c}\right) e_{\mu}^{c}
$$

Then the part of $S_{5}$ which is zeroth-order in $\hbar$ is 


$$
\begin{aligned}
S_{5}= & \frac{1}{4} \int d^{5} x \sqrt{g}\left[\frac{1}{\kappa^{2}} g^{\mu \nu} \partial_{5} e_{\mu}^{a} \partial_{5} e_{\nu}^{a}+4\left(\frac{1}{\kappa^{2}} \bar{R}_{\mu}^{a} \bar{R}_{\sigma}^{a} g^{\mu \sigma}-\lambda \bar{R}+\kappa^{2} \lambda^{2}\right)\right. \\
& \left.+(\Omega \wedge e)_{\mu \nu}^{a}(\Omega \wedge e)_{\rho \sigma}^{b} g^{\nu \sigma}\left(\delta_{a b} g^{\mu \rho}+2 e_{a}^{\mu} e_{b}^{\rho}\right)+O(\sqrt{\hbar})\right]
\end{aligned}
$$

where $\bar{R}=d \bar{\omega}+\bar{\omega} \wedge \bar{\omega}$. Integrating over $\Omega$ cancels a factor $\sqrt{G_{\omega}}$ in the measure, leaving

$$
\begin{aligned}
P[\lambda] & =\frac{1}{Z} \int D e\left(\mathbf{x}, t_{5}\right) \sqrt{G_{e}} e^{-S_{5}[e, \lambda] / \hbar} \\
S_{5}[e, \lambda] & =\frac{1}{4} \int d^{5} x \sqrt{g}\left[\frac{1}{\kappa^{2}} g^{\mu \nu} \partial_{5} e_{\mu}^{a} \partial_{5} e_{\nu}^{a}+4\left(\frac{1}{\kappa^{2}} \bar{R}_{\mu}^{a} \bar{R}_{\sigma}^{a} g^{\mu \sigma}-\lambda \bar{R}+\kappa^{2} \lambda^{2}\right)\right]
\end{aligned}
$$

The Baum-Hawking mechanism is not immediately apparent in (26), simply because $S_{5}$ is bounded, and in fact vanishes when evaluated at the classical solution $S_{5}[\bar{e}, \lambda]=0$. So if there is a peak in $P[\lambda]$ at $\lambda=0^{+}$, it would have to come from quantum fluctuations. Fortunately this is just what happens, as we now show at one-loop level. Quantum gravity is perturbatively non-renormalizable; this situation is unchanged by stabilization, so to do a perturbative evaluation of (26) we must introduce a short-distance cutoff $\Lambda^{-1}$, presumably on the order of the Planck length, where some new physics or fundamental granularity of spacetime comes into play. Expanding the action in (26) only up to terms quadratic in the fluctuations

$$
S_{5} \approx \int d^{5} x \delta e M[\bar{e}] \delta e
$$

and integrating over the fluctuations yields the one-loop result

$$
P(\lambda) \approx \operatorname{det}^{-1 / 2}[M]
$$

where the determinant needs to be regulated by, e.g., the heat-kernel technique. As is well-known, the regulated determinant can be expanded in powers of the background Riemann tensor [15, 16]

$$
P(\lambda) \approx \exp \left[c_{0} \int d^{5} x \sqrt{\bar{g}}+c_{1} \int d^{5} x \sqrt{\bar{g}} R(\bar{g})+\ldots\right]
$$

where $c_{0}, c_{1}$ are cutoff-dependent constants. Because of the non-renormalizability of quantum gravity we are not entitled to drop such constants and just concentrate on the cutoff-independent terms. The cutoff at the Planck length is a true physical scale where new physics is encountered, perhaps in the form of strings or a fundamental lattice. The largest powers of $\Lambda$ and $1 / \lambda$ are in the first term of the expansion, and $c_{0}$ is most easily calculated by setting $\lambda=0$, and calculating the determinant 
around flat space. Expanding $S_{5}$ around flat space, $e_{\mu a}=\delta_{\mu a}+\delta e_{\mu a}$, we find for the part quadratic in fluctuations

$$
\begin{aligned}
S_{5}= & \frac{1}{\kappa^{2}} \int d^{5} x \delta e_{\mu \nu}\left[\frac{1}{4}\left(-\partial_{5}^{2}\right) \delta_{\mu \alpha} \delta_{\nu \beta}+\left(\partial_{\mu} \partial^{\mu}\right)^{2}\left\{P^{(2)}+4 P^{(0 s)}\right\}_{\mu \nu \alpha \beta}\right] \delta e_{\alpha \beta} \\
= & \frac{1}{\kappa^{2}} \int d^{5} x \delta e_{\mu \nu}\left\{\left[\frac{1}{4}\left(-\partial_{5}^{2}\right)+\left(\partial_{\mu} \partial^{\mu}\right)^{2}\right] P^{(2)}+\left[\frac{1}{4}\left(-\partial_{5}^{2}\right)+4\left(\partial_{\mu} \partial^{\mu}\right)^{2}\right] P^{(0 s)}\right. \\
& \left.+\frac{1}{4}\left(-\partial_{5}^{2}\right)\left(\mathbf{1}_{A}+P^{(1)}+P^{(0 w)}\right)\right\}_{\mu \nu \alpha \beta} \delta e_{\alpha \beta}
\end{aligned}
$$

where

$$
\mathbf{1}_{A} \equiv \frac{1}{2}\left(\delta_{\mu \alpha} \delta_{\nu \beta}-\delta_{\mu \beta} \delta_{\nu \alpha}\right)
$$

and $P^{(2)}, P^{(1)}, P^{(0 s)}, P^{(0 w)}$ are spin projection operators introduced by van Nieuwenhuizen [17]. Using the heat-kernel prescription

$$
\begin{aligned}
\operatorname{lndet}[M] & =-\int_{\kappa^{2} / \Lambda^{4}}^{\infty} \frac{d s}{s} \int d^{5} x \sqrt{\bar{g}} \operatorname{Tr}[K(x, x, s)] \\
\frac{d}{d s} K & =-M K
\end{aligned}
$$

a short computation finds that

$$
c_{0}=\frac{21}{12 \sqrt{\pi}}\left(\frac{\Gamma\left(\frac{1}{4}\right)}{4 \pi}\right)^{4} \Lambda^{6}+\frac{5}{2 \sqrt{\pi}} \delta^{4}(0) \Lambda^{2}=c \Lambda^{6}
$$

where the delta-function is also understood to be regulated by the short-distance cutoff, i.e. $\delta^{4}(0) \approx \Lambda^{4}$. The precise value of $c$ is not really important; all we need is the fact that it is positive. Note that gauge-fixing is unnecessary in this calculation, since $S_{5}$ is only invariant under $t_{5}$-independent transformations.

For finite $\lambda$ we have

$$
\int d^{5} x \sqrt{\bar{g}}=\frac{3 T}{8 G^{2} \lambda^{2}}
$$

where $T$ is the extension of the $t_{5}$ axis (note that $t_{5}$ has units of length squared), and therefore, to leading order in $\Lambda$ and $1 / \lambda$,

$$
P(\lambda) \approx \exp \left[\frac{3 c \Lambda^{6} T}{8 G^{2} \lambda^{2}}\right]
$$

This expression has been derived for $\lambda>0$. For $\lambda<0$ there are other solutions of the Einstein equations, representing compact manifolds of non-spherical topology,

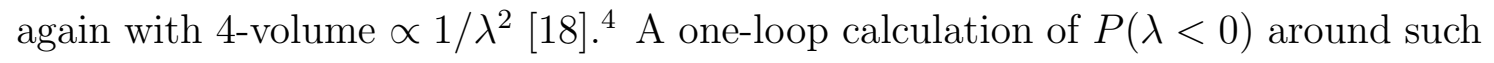

\footnotetext{
${ }^{4}$ There are also noncompact solutions for $\lambda<0$; however (by assumption) the functional integral is restricted to metrics representing compact 4-manifolds.
} 
backgrounds will produce an expression similar to (35), differing only by replacement of the factor $3 / 8$ in the exponent by some other positive constant.

Equation (35) is the promised result. As in the Baum-Hawking expression (2), there is clearly an infinite peaking in the probability distribution of the regulated theory at $\lambda \rightarrow 0$, which is offered here as an explanation for the vanishing of the cosmological constant. Unlike the Baum-Hawking expression (2), eq. (35) is a oneloop result, obtained from a well-defined functional integral with a gravitational action which is bounded from below.

\section{References}

[1] E. Baum, Phys. Lett. B133 (1983) 185.

[2] S. Hawking, Phys. Lett. B134 (1984) 403.

[3] S. Coleman, Nucl. Phys. B310 (1988) 643.

[4] G. Gibbons, S. Hawking, and M. Perry, Nucl. Phys. B138 (1978) 141.

[5] G. Horowitz, Phys. Rev. D31 (1985) 1169.

[6] J. Polchinski, Phys. Lett. B219 (1989) 251.

[7] F. David, Mod. Phys. Lett. A5 (1990) 1019; Nucl. Phys. B348 (1991) 507; P. Silvestrov and A. Yelkhovsky, Phys. Lett. B251 (1990) 525.

[8] J. Greensite and M. Halpern, Nucl. Phys. B242 (1984) 167.

[9] E. Marinari and G. Parisi, Phys. Lett. B240 (1990) 375; A. Migdal and M. Karliner, Mod. Phys. Lett. A5 (1990) 2565; J. Ambjorn, J. Greensite, and S. Varsted, Phys. Lett. B249 (1990) 411; J. Ambjorn and J. Greensite, Phys. Lett. B254 (1991) 66; J. Miramontes, J. Guillen and M. Vozmediano, Phys. Lett. B253 (1991) 38; J. Gonzalez and M. Vozmediano, Phys. Lett. B258 (1991) 55.

[10] J. Greensite, Nucl. Phys. B361 (1991) 729.

[11] J. Greensite, "Stabilized Quantum Gravity: Stochastic Interpretation and Numerical Simulation", San Francisco preprint SFSU-TH-92/1, hepth@xxx/9205006.

[12] A. Carlini and M. Martellini, Phys. Lett. B276 (1992) 36. 
[13] A. Giveon, M. Halpern, E. Kiritsis, and N. Obers, Nucl. Phys. B357 (1991) 655.

[14] C. Misner, K. Thorne, and J. Wheeler, Gravitation (W. H. Freeman, San Francisco, 1973), p. 471.

[15] B. DeWitt, Dynamical Theory of Groups and Fields (Gordon and Breach, New York, 1965) p. 231.

[16] P. Gilkey, J. Diff. Geom. 10 (1975) 601.

[17] P. van Nieuwenhuizen, Nucl. Phys. B60 (1973) 478.

[18] S. Hawking, Nucl. Phys. B144 (1978) 349. 